\title{
Mutation of ARHGAP9 in patients with coronary spastic angina
}

\author{
Mikito Takefuji ${ }^{1,2,11}$, Hiroyuki Asano ${ }^{2,11}$, Kazutaka Mori ${ }^{1,2}$, Mutsuki Amano ${ }^{1}$, Katsuhiro Kato ${ }^{1,2}$, \\ Takashi Watanabe ${ }^{1,3}$, Yasuhiro Morita ${ }^{1,2}$, Akira Katsumi ${ }^{4}$, Toshiki Itoh ${ }^{5}$, Tadaomi Takenawa ${ }^{5}$, \\ Akihiro Hirashiki $^{2}$, Hideo Izawa ${ }^{6}$, Kozo Nagata $^{7}$, Haruo Hirayama ${ }^{8}$, Fumimaro Takatsu ${ }^{9}$, Tomoki Naoe ${ }^{4}$, \\ Mitsuhiro Yokota ${ }^{10}$ and Kozo Kaibuchi ${ }^{1}$
}

Coronary artery spasm has an important function in the etiology of variant angina and other acute coronary syndromes. Abnormal activation of Rho-family GTPases has been observed in cardiovascular disorders, but the function of genetic variability in Rho-family GTPases remains to be evaluated in cardiovascular disorders. We examined the genetic variability of Rho-family GTPases and their regulators in coronary artery spasm. We performed a comprehensive candidate gene analysis of 67 single nucleotide polymorphisms with amino-acid substitution in Rho-family GTPases and their regulators in 103 unrelated Japanese patients with acetylcholine-induced coronary artery spasm and 102 control Japanese subjects without acetylcholine-induced coronary artery spasm. We noted an association of the single nucleotide polymorphism of ARHGAP9 (rs11544238, Ala370Ser) with coronary artery spasm (odds ratio=2.67). We found that ARHGAP9 inactivated Rac as RacGAP and that the mRNA level of ARHGAP9 was strongly detected in hematopoietic cells. ARHGAP9 negatively regulated cell migration. The Ala370Ser polymorphism counteracted ARHGAP9-reduced cell migration, spreading and adhesion. The Ala370Ser polymorphism in the ARHGAP9 gene is associated with coronary artery spasm. These data suggest that the polymorphism of ARHGAP9 has a critical function in the infiltration of hematopoietic cells into the endothelium and inflammation leading to endothelial dysfunction. Journal of Human Genetics (2010) 55, 42-49; doi:10.1038/jhg.2009.120; published online 13 November 2009

Keywords: ARHGAP9; coronary artery spasm; Rho-family GTPases; SNP

\section{INTRODUCTION}

Coronary artery spasm has an important function in the etiology of Prinzmetal variant angina and other acute coronary syndromes. ${ }^{1,2}$ Although the physiological and genetic risk factors for coronary spastic angina (CSA) have been reported, ${ }^{3}$ the precise mechanisms of CSA remain largely unknown. Some reports indicated that coronary artery spasm is related to the extent of atherosclerotic changes 4 and endothelial dysfunction. ${ }^{5}$ Endothelial dysfunction was initially characterized by impaired vasodilation to specific stimuli, such as acetylcholine that can induce vasodilation by release of nitric oxide from the endothelium. ${ }^{6,7}$ The broader understanding of endothelial dysfunction includes proinflammatory and prothrombotic states of the endothelium. ${ }^{8}$ Several investigators have reported that inflammatory markers are involved in coronary artery spasm. ${ }^{9-11}$ Taken together, these observations suggest that coronary artery spasm is related to inflammation at the endothelium leading to endothelial dysfunction.

The prevalence of coronary artery spasm has been shown to be higher in Japanese patients than in the Caucasian population. ${ }^{12}$ Conversely, the prevalence of obstructive coronary artery disease is lower among the Japanese than among the Caucasians. ${ }^{13}$ This racial difference suggests that genetic factors contribute to the pathophysiology of coronary artery spasm and that genetic epidemiological studies have been performed on Japanese subjects. ${ }^{14}$

Abnormal activation of the Rho-family GTPases, including Rho, Rac and Cdc42, has been observed in cardiovascular disorders such as hypertension and atherosclerosis. We previously reported that Rhokinase/ROCK, an effector of RhoA, phosphorylates and inactivates myosin phosphatase, and thereby increases the phosphorylation of myosin light chain followed by smooth muscle contraction. ${ }^{15,16}$

${ }^{1}$ Department of Cell Pharmacology, Graduate School of Medicine, Nagoya University, Nagoya, Japan; ${ }^{2}$ Department of Cardiology, Graduate School of Medicine, Nagoya University, Nagoya, Japan; ${ }^{3}$ Institute for Advanced Research, Nagoya University, Nagoya, Japan; ${ }^{4}$ Department of Hematology and Oncology, Graduate School of Medicine, Nagoya University, Nagoya, Japan; ${ }^{5}$ Department of Biochemistry and Molecular Biology, Graduate School of Medicine, Kobe University, Kobe, Japan; ${ }^{6}$ Department of Internal Medicine, Fujita Health University Hospital, Nagoya, Japan; ${ }^{7}$ Department of Medical Technology, School of Health Sciences, Nagoya University, Nagoya, Japan; ${ }^{8}$ Department of Cardiology, Nagoya Daini Red Cross Hospital, Nagoya, Japan; ${ }^{9}$ Division of Cardiology, Kosei Hospital, Anjo, Japan and ${ }^{10}$ Department of Genome Science, School of Dentistry, Aichi-Gakuin University, Nagoya, Japan

${ }^{11}$ These authors should be regarded as joint first authors.

Correspondence: Professor K Kaibuchi, Department of Cell Pharmacology, Graduate School of Medicine, Nagoya University, 65 Tsurumai, Showa-ku, Nagoya 466-8550, Japan. E-mail: kaibuchi@med.nagoya-u.ac.jp

Received 30 June 2009; revised 19 October 2009; accepted 19 October 2009; published online 13 November 2009 
The RhoA/Rho-kinase pathway increases the sensitivity of vascular smooth muscle toward $\mathrm{Ca}^{2+}$ in CSA and hypertension. ${ }^{17}$ Rac generally regulates actin polymerization and membrane protrusion at the front leading edge during cell migration, whereas Rho regulates contraction at the rear. ${ }^{18}$ This cooperation of Rac and Rho mediates the migration of various types of cell, such as leukocytes and monocytes, at the atherosclerotic site. ${ }^{19}$

The Rho-family GTPases cycle between an inactive GDP-bound state and an active GTP-bound state. ${ }^{20}$ There are three major classes of regulators of the Rho-family GTPases: guanine nucleotide exchange factors (GEFs) facilitate the exchange of GDP for GTP to activate Rhofamily GTPases; GTPase-activating proteins (GAPs) stimulate their intrinsic GTPase activity to turn off Rho-family GTPases; and guanine nucleotide dissociation inhibitors control cycling of Rho-family GTPases between membranes and cytosol.

Although many reports have shown that the RhoA/Rho-kinase pathway is related to CSA, the involvement of other Rho family members and their regulators in CSA remains unknown. In light of these observations, we examined the function of the genetic variability of the Rho family and their regulatory genes in coronary artery spasm.

\section{MATERIALS AND METHODS}

\section{Study subjects}

The study population comprised 205 unrelated Japanese individuals (163 men, 42 women) who underwent coronary angiography after intracoronary injection of acetylcholine to diagnose coronary artery spasm between July 1994 and December 2001. They had no history of myocardial infarction and effort angina pectoris.

In this study, all medication was withdrawn at least $48 \mathrm{~h}$ before coronary angiography. Coronary artery spasm was induced as previously described. ${ }^{21}$ In brief, after baseline angiography of the left and right coronary arteries, two doses (50 and $100 \mu \mathrm{g}$ ) of acetylcholine were injected sequentially into the left coronary artery and angiography was performed again.

In 103 patients (82 men, 21 women), coronary artery spasms were angiographically documented after intracoronary injection of acetylcholine. Coronary artery spasm was defined as either total or $\geqslant 90 \%$ occlusion of the epicardial coronary arteries associated with chest pain and ischemic ST segment elevation. In 102 control subjects ( 81 men, 21 women), coronary artery spasms were not documented after intracoronary injection of acetylcholine.

Patients with acetylcholine-induced coronary artery spasm and subjects without acetylcholine-induced coronary artery spasm either had or did not have conventional risk factors for coronary artery disease, including regular cigarette smoking ( $\geqslant 10$ cigarettes daily), hypertension (systolic blood pressure $\geqslant 140 \mathrm{~mm} \mathrm{Hg}$ and/or diastolic blood pressure $\geqslant 90 \mathrm{~mm} \mathrm{Hg}$ ), diabetes mellitus (fasting blood glucose $\geqslant 6.93 \mathrm{mmoll}^{-1}$ and/or hemoglobin A1C $\geqslant 6.5 \%$ ), hyperlipidemia (serum total cholesterol $\geqslant 5.72 \mathrm{mmoll}^{-1}$ ) and hyperuricemia (serum uric acid $\geqslant 0.46 \mathrm{mmoll}^{-1}$ for men or $\geqslant 0.33 \mathrm{mmoll}^{-1}$ for women; Table 2). The study protocol complies with the Declaration of Helsinki Principles and was approved by the Committees on the Ethics of Human Research of Nagoya University Graduate School of Medicine, Nagoya Daini Red Cross Hospital and Kosei Hospital. Written informed consent was obtained from all participants.

\section{Selection of polymorphisms}

With the use of public databases, including PubMed and/or GeneCards, we selected 67 polymorphisms with amino-acid substitutions in candidate genes of small GTPases, Rho-family GEFs and Rho-family GAPs (Table 1).

\section{Genotyping of polymorphisms}

Venous blood $(7 \mathrm{ml})$ was collected from each subject and genomic DNA was isolated with a kit (Qiagen, Chatsworth, CA, USA). Genotypes of polymorphisms were determined with a fluorescence- or colorimetry-based allele-specific DNA primer-probe assay system as previously described (Supplementary Table 2). ${ }^{22}$

\section{Statistical analysis}

Quantitative clinical data were compared between patients with coronary artery spasm and control subjects using the unpaired Student's $t$-test. Qualitative data were compared by the $\chi^{2}$-test. Allele frequencies were estimated by the gene counting method, and the $\chi^{2}$-test was used to identify significant departures from the Hardy-Weinberg equilibrium. We performed a multivariable logistic regression analysis to adjust for risk factors, with coronary artery spasm as a dependent variable and independent variables including age, body mass index, smoking status, metabolic variables (history of hypertension, diabetes mellitus, hypercholesterolemia and hyperuricemia) and genotype of each polymorphism. There were three genotypes for each polymorphism: common homozygote, heterozygote and variant homozygote. A dominant genetic model was defined as a model comprising two groups: common homozygotes and the combined group of heterozygotes and variant homozygotes. A recessive model was also defined as a model of two groups: variant homozygotes and the combined group of common homozygotes and heterozygotes. An additive model was defined as a model consisting of three genotype groups. Each genotype was assessed according to dominant, recessive and additive genetic models, and the $P$-value (Wald test), odds ratio and 95\% confidence interval (profile likelihood ratio method) were calculated. All analyses were performed using the Statistical Package for Social Science (SPSS, Chicago, IL, USA) version 14.0. Statistical significance was examined by two-sided tests. $P$-value less than 0.05 was considered to be statistically significant.

\section{Materials and chemicals}

We obtained human ARHGAP9 cDNA from ATCC (Manassa, VA, USA), monoclonal anti-GFP antibody from Roche Diagnostics (Mannheim, Germany), anti-CD3 antibody from BD Biosciences (San Jose, CA, USA) and antiCD14 antibody from Caltag Laboratories (Burlingame, CA, USA). Anti-HA monoclonal antibody (12CA5) was prepared from mouse hybridoma cells. Other materials and chemicals were obtained from commercial sources. ARHGAP9 fragments were amplified by PCR and subcloned into pEGFP (Takara Bio, Otsu, Japan) plasmids.

\section{Reverse transcriptase-PCR}

We evaluated mRNA expression in cells by reverse transcriptase (RT)-PCR with the RNeasy Protect kit (Qiagen) and SuperScript First-Strand Synthesis System (Invitrogen, Carlsbad, CA, USA). Human lymphocytes were isolated from whole blood of healthy volunteers with Ficoll-Paque Plus (GE Healthcare, Little Chalfont, UK). CD3-positive cells (T cells) and CD14-positive cells (monocytes) were gathered from isolated lymphocytes with FACS Aria Ver2.0 Diva4.1 (BD Biosciences). The primers were as follows: forward, 5'-TCCGAT GGGAGGTATGTGTT- $3^{\prime}$ and reverse, $5^{\prime}$-ATCTGAGTGTGCTATCACCCTG- ${ }^{\prime}$ for human ARHGAP9 and forward, 5'-CTTCACCACCATGGAGAAGG-3' and reverse, 5'-TGAAGTCAGAGGAGACCACC-3' for human glyceraldehyde 3-phosphate dehydrogenase (GAPDH).

We collected blood samples from healthy volunteers, and total RNA was extracted from blood samples with the QIAmp RNA blood kit (Qiagen). The study protocol complies with the Declaration of Helsinki Principles and was approved by the Committees on the Ethics of Human Research of Nagoya University Graduate School of Medicine. Real-time RT-PCR analysis was performed as previously described. ${ }^{23}$ The results are representative of three independent experiments.

\section{Pull-down assay}

COS7 cells (35-mm dish) were transfected with EGFP-ARHGAP9-GAP or EGFP-ARHGAP9- $\Delta \mathrm{N}$, and HA-RhoA, EGFP-Rac1, EGFP-Rac2 or EGFPCdc42 using Lipofectamine (Invitrogen). After an 18-h incubation, the cells were lysed in $500 \mu \mathrm{l}$ of lysis buffer ( $50 \mathrm{~mm}$ Tris- $\mathrm{HCl}$, at pH 7.0, 1 mм EGTA, $10 \mathrm{~mm} \mathrm{MgCl}_{2}, 500 \mathrm{~mm} \mathrm{NaCl}, 0.5 \% \mathrm{NP}-40,0.1 \mathrm{~mm}$ ( $p$-amidinophenyl)methanesulfonyl fluoride, $2.5 \mu \mathrm{g} \mathrm{ml}^{-1}$ aprotinin, $2.5 \mu \mathrm{g} \mathrm{ml}^{-1}$ leupeptin) containing $15 \mu \mathrm{g}$ GST-Rho binding domain of Rhotekin (GST-RBD) or GST-Cdc42/Rac interactive binding region of PAK1 protein (GST-PAK-CRIB). The lysates were centrifuged, and the supernatants were incubated with Glutathione-Sepharose $4 \mathrm{~B}$ beads (GE Healthcare) for $30 \mathrm{~min}$ at $4{ }^{\circ} \mathrm{C}$. The beads were washed with lysis buffer and then eluted with a SDS sample buffer. The eluates were subjected to 
Table 1 The polymorphisms of small GTPases, Rho-family GEFs and Rho-family GAPs examined for association with CSA

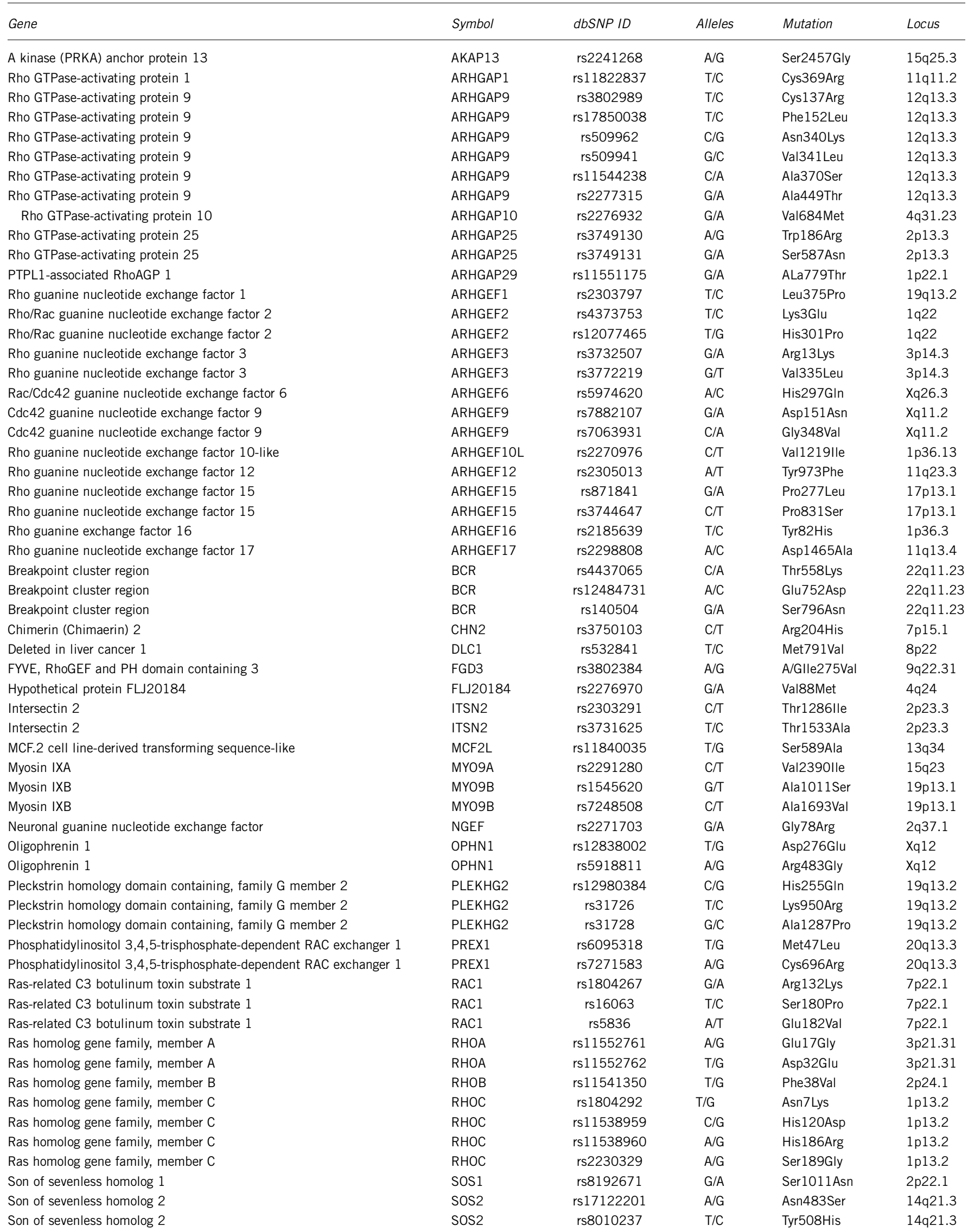


Table 1 Continued

\begin{tabular}{|c|c|c|c|c|c|}
\hline Gene & Symbol & $d b S N P I D$ & Alleles & Mutation & Locus \\
\hline Triple functional domain & TRIO & rs 16903367 & $\mathrm{G} / \mathrm{T}$ & Glu348Asp & $5 p 15.2$ \\
\hline Triple functional domain & TRIO & rs 16903474 & $A / G$ & Thr1613Ala & $5 p 15.2$ \\
\hline Vav 2 oncogene & VAV2 & rs602990 & $\mathrm{C} / \mathrm{T}$ & Val584Met & $9 q 34.2$ \\
\hline Vav 3 oncogene & VAV3 & rs7528153 & $\mathrm{T} / \mathrm{A}$ & Ser298Thr & $1 \mathrm{p} 13.3$ \\
\hline
\end{tabular}

The dpSNP IDs were obtained from the Entrez SNP website (http://www.ncbi.nlm.nih.gov/SNP/). Mutation shows ancestral amino acid, amino-acid position and changed amino acid, in order starting from the left.

Table 2 Characteristics of study subjects

\begin{tabular}{lcc}
\hline & \multicolumn{2}{c}{ ACh spasm provocation test } \\
\cline { 2 - 3 } Characteristic & Spasm (-) & Spasm (+) \\
\hline$n$ (male/female) & $102(81 / 21)$ & $103(82 / 21)$ \\
Age (years) & $57.3 \pm 8.8$ & $57.9 \pm 9.1$ \\
BMI (kg m ${ }^{-2}$ ) & $23.7 \pm 2.8$ & $23.1 \pm 2.8$ \\
Smoker (\%) & 68.6 & 72.8 \\
Hypertension (\%) & 80.4 & $62.1^{*}$ \\
Type II diabetes (\%) & 21.6 & $8.7^{*}$ \\
Hyperlipidemia (\%) & 49.0 & 36.9 \\
Hyperuricemia (\%) & 24.5 & $12.6^{*}$ \\
\hline
\end{tabular}

Abbreviation: ACh, acetylcholine; BMI, body mass index.

Quantitative data are means \pm standard deviation.

${ }^{*} P<0.05$ vs others.

SDS-polyacrylamide gel electrophoresis, followed by immunoblotting with an anti-GFP antibody or anti-HA antibody. The results are representative of three independent experiments.

\section{Three-dimensional cell migration assay}

A three-dimensional cell migration (the modified Boyden chamber) assay was performed using Transwell (Coaster, Cambridge, MA, USA) 24-well tissue culture plates composed of a polycarbonate membrane containing $8 \mu \mathrm{m}$ pores, as previously described. ${ }^{24}$ Vero cells were transfected with EGFP-GST or EGFPARHGAP9-FL fragments using Lipofectamine 2000 (Invitrogen). At $18 \mathrm{~h}$ after transfection, $1 \times 10^{4}$ Vero cells in $500 \mu \mathrm{l}$ of serum-free medium containing $0.1 \%$ bovine serum albumin (BSA) were seeded on the upper chamber of the Transwell precoated with $10 \mu \mathrm{g} \mathrm{ml}^{-1}$ of fibronectin (Sigma-Aldrich, St Louis, MO, USA). After a 3-h incubation, the cells that had migrated to the lower side of the membrane were fixed with $3.0 \%$ formaldehyde in phosphate-buffered saline (PBS) and then treated with PBS containing $0.2 \%$ Triton X-100 and $2 \mathrm{mg} \mathrm{ml}^{-1}$ BSA. The fixed cells were stained with $100 \mu \mathrm{g} \mathrm{ml}^{-1}$ of Hoechst 33342 (Sigma-Aldrich). The number of Hoechst 33342 and EGFP-positive cells in 20 randomly chosen fields in each Transwell was counted under a Zeiss axiophoto microscope (Carl Zeiss, Oberkochen, Germany). The results are representative of three independent experiments at $\times 200$ magnification $(n>20$ in each experiment).

\section{Cell spreading assay}

HeLa cells were transfected with EGFP-ARHGAP9-FL fragments using Lipofectamine (Invitrogen) and cultured in serum-free medium containing $0.1 \%$ BSA. RAW264 cells were transfected with EGFP-ARHGAP9-FL using Nucleofector kit (Amaxa Biosystems, Cologne, Germany). At $18 \mathrm{~h}$ after transfection, the cells were replated on coverslips precoated with $10 \mu \mathrm{g} \mathrm{m}^{-1}$ fibronectin. After a 1-h incubation, the cells were washed with PBS twice and fixed as described above. The fixed cells were stained with tetramethylrhodamine B isothiocyanate-phalloidin to measure the area of spread cells using the software
LSM 510 (Carl Zeiss). We defined HeLa cells $\left(\geqslant 400 \mu \mathrm{m}^{2}\right)$ and RAW264 cells $\left(\geqslant 40 \mu \mathrm{m}^{2}\right)$ as spread cells. The results are representative of three independent experiments ( $n>200$ in each experiment).

\section{Cell-adhesion assay}

The cell-adhesion assays were performed as described elsewhere. ${ }^{25}$ Jurkat cells were transfected with EGFP-ARHGAP9-FL using the Nucleofector kit (Amaxa Biosystems) and incubated in serum-free medium for 24-h after transfection. GFP-positive cells (5000) were plated on each coverslip precoated with $10 \mu \mathrm{g} \mathrm{ml}^{-1}$ fibronectin in 24-well plates. After a 30-min incubation, the cells were washed with PBS and fixed as described above. The number of GFP-positive cells was counted. The results are representative of three independent experiments.

\section{RESULTS}

\section{Comprehensive candidate gene analysis}

As a first step, we searched for polymorphisms with amino-acid substitution among the Rho family members and their regulators (more than 140 different genes), and identified 67 polymorphisms (Table 1). The study population comprised 205 unrelated Japanese individuals who had no history of myocardial infarction and effort angina pectoris. After we confirmed that the coronary arteries appeared normal or exhibited no significant organic stenosis ( $<25 \%$ luminal diameter) in all subjects by a coronary angiography, we performed a coronary angiography after intracoronary injection of acetylcholine. The genotypic distributions of the polymorphisms between 103 patients with both acetylcholine-induced coronary artery spasm and angina pectoris at rest and 102 control subjects without acetylcholine-induced coronary artery spasm were in Hardy-Weinberg equilibrium (Supplementary Table 1). The characteristics of 103 patients and 102 control subjects are shown in Table 2. On the basis of multivariate logistic regression analysis with adjustment for age, body mass index, and the prevalence of smoking, hypertension, diabetes mellitus, hyperlipidemia and hyperuricemia, the Ala370Ser and Ala449Thr polymorphisms in the ARHGAP9 gene and the Val1219Ile polymorphism in the AUHGEF10L gene were associated with a significant risk of coronary artery spasm (Table 3 ). However, the Ala370Ser and Ala449Thr polymorphisms were in linkage disequilibrium and the minor allele frequency of the Val1219Ile polymorphism was especially low (Supplementary Table 1). Subjects with the Ala370Ser polymorphism in the ARHGAP9 gene were more susceptible to coronary artery spasm than the corresponding individuals with the Ala449Thr polymorphism. Therefore, we regarded the Ala370Ser polymorphism in ARHGAP9 as a coronary artery spasm susceptibility polymorphism.

\section{Gene expression of ARHGAP9}

To examine the expression of ARHGAP9 in the cells related to coronary artery spasm, we evaluated the mRNA levels of ARHGAP9 
Table 3 Multivariate logistic regression analysis of polymorphisms associated with CSA

\begin{tabular}{lcccccccc}
\hline & & \multicolumn{7}{c}{ Minor } \\
Gene & dbSNPID & Alleles & Mutation & allele & model & OR & P-value \\
\hline ARHGAP9 & rs11544238 & C/A & Ala370Ser & A & Additive & 1.77 & 0.006 \\
ARHGAP9 & rs11544238 & C/A & Ala370Ser & A & Dominant & 2.67 & 0.004 \\
ARHGAP9 & rs2277315 & G/A & Ala449Thr & A & Additive & 1.54 & 0.031 \\
ARHGAP9 & rs2277315 & G/A & Ala449Thr & A & Dominant & 2.25 & 0.011 \\
ARHGEF10L & rs2270976 & C/T & Val1219lle & T & Dominant & 0.45 & 0.043 \\
\hline
\end{tabular}
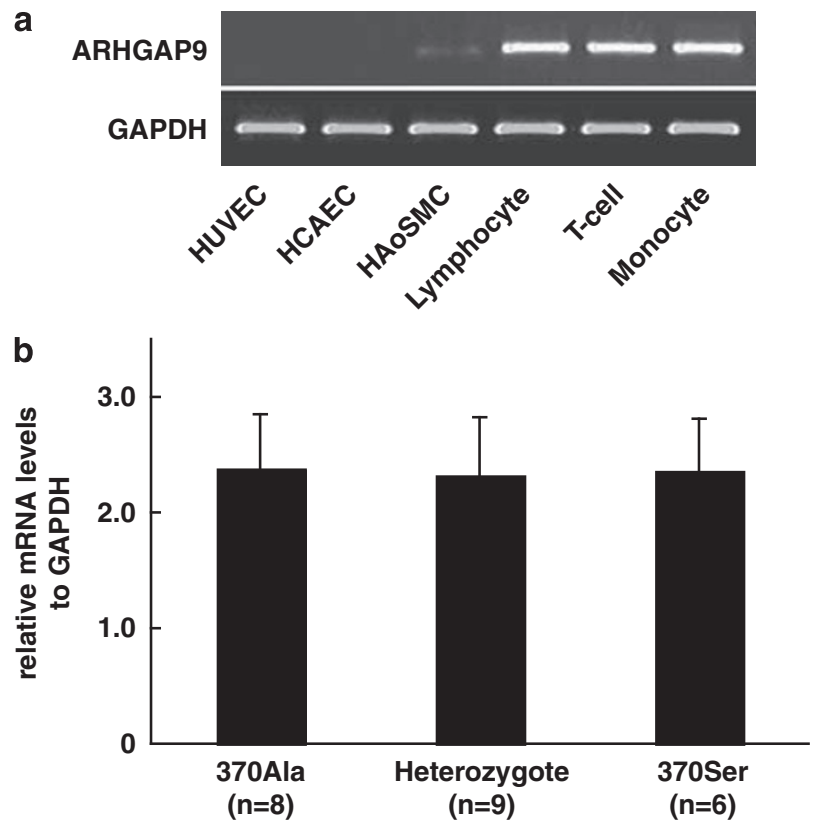

Figure 1 Detection of specific mRNA for ARHGAP9. (a) Total RNA was extracted from normal human umbilical vein endothelial cells (HUVECs), normal human coronary artery endothelial cells (HCAECs), normal human aortic smooth muscle cells (HAoSMCs), human blood lymphocytes, human blood T cells (CD3-positive lymphocyte) and human blood monocytes (CD14-positive lymphocyte). Gene expression was evaluated by reverse transcriptase (RT)-PCR analysis. (b) Specific mRNAs for ARHGAP9 and glyceraldehyde 3-phosphate dehydrogenase (GAPDH) were detected in blood lymphocytes of subjects with ARHGAP9 370Ala, ARHGAP9 370 Ser or ARHGAP9 heterozygote by real-time RT-PCR analysis. The ratio of ARHGAP9 to GAPDH is shown.

by RT-PCR in normal human umbilical vein endothelial cells (HUVECs), normal human coronary artery endothelial cells (HCAECs), normal human aortic smooth muscle cells (HAoSMCs), human lymphocytes, human CD3-positive lymphocytes ( $\mathrm{T}$ cells) and human CD14-positive cells (monocytes; Figure 1a). The specific PCR product for the ARHGAP9 gene was detected strongly in T cells and monocytes, and slightly in HAoSMCs. The product was hardly detected in HUVECs and HCAECs.

We next examined whether the Ala370Ser polymorphism in the ARHGAP9 gene influences its mRNA expression (Figure 1b). mRNA was prepared from white blood cells of subjects with the ARHGAP9 370Ala, ARHGAP9 370Ser or ARHGAP9 heterozygote. There were no significant differences in the mRNA expression of ARHGAP9 in blood samples among subjects with the ARHGAP9 370Ala, ARHGAP9 370Ser and ARHGAP9 heterozygotes.

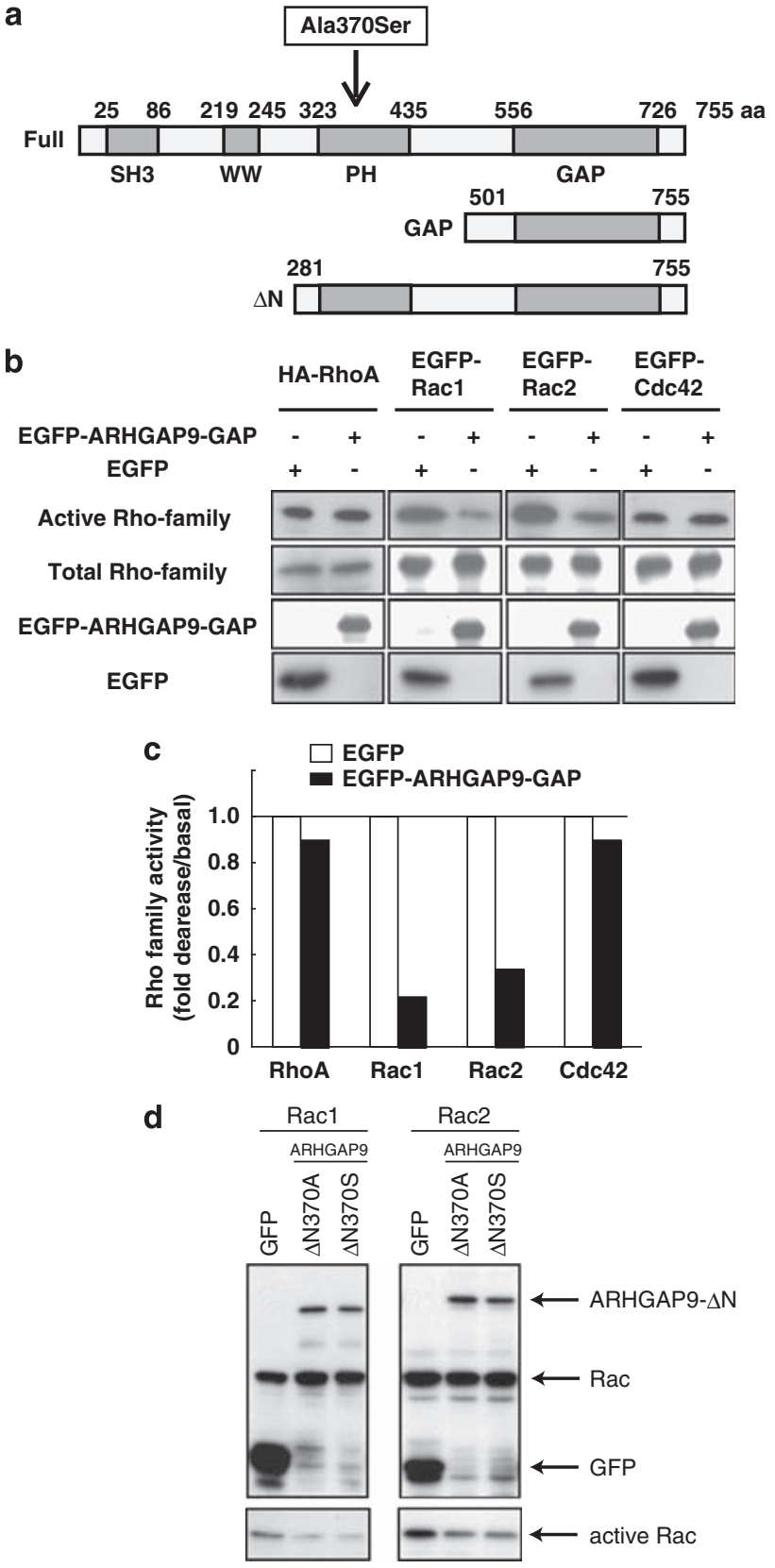

Figure 2 GTPase-activating protein (GAP) activity of EGFP-ARHGAP9-GAP to Rho-family GTPases in COS7 cells. (a) The domain structure and deletion constructs of ARHGAP9 are represented. (b) Cells transfected with the indicated constructs were incubated with GST-Rho binding domain of Rhotekin (GST-RBD) or GST-Cdc42/Rac interactive binding region of PAK1 protein (GST-PAK-CRIB) to precipitate the GTP form of Rho family GTPases. The amounts of GTP-bound Rho family GTPases, total Rho family GTPases, EGFP-ARHGAP9 and EGFP were determined by immunoblotting with HA or GFP antibody. (c) The ratio of GTP-bound Rho family GTPases to total Rho family GTPases is shown. (d) Cells transfected with the indicated constructs were incubated with GST-PAK-CRIB to precipitate the GTP form of Rac1 or Rac2. The amounts of GTP-bound Rac, total Rac, ARHGAP9 and GFP were determined by immunoblotting with the GFP antibody.

\section{Function of ARHGAP9}

Figure 2a shows the protein domains of ARHGAP9 and the aminoacid exchange of ARHGAP9 (Ala370Ser) in the pleckstrin homology $(\mathrm{PH})$ domain. It has been reported that ARHGAP9 enhances GTP 
hydrolysis activity of Racl and Cdc42 in vitro. ${ }^{25}$ To investigate the GAP activity of ARHGAP9 toward the Rho-family GTPases in vivo, we transfected EGFP-ARHGAP9-GAP (aa 501-755) and HA-RhoA, EGFP-Rac1, EGFP-Rac2 or EGFP-Cdc42 into COS7 cells and monitored its GAP activity by a pull-down assay (Figures $2 \mathrm{~b}, \mathrm{c}$ ). The expression of EGFP-ARHGAP9-GAP reduced the amount of GTPbound EGFP-Rac1 and EGFP-Rac2, but not that of EGFP-Cdc42 or HA-RhoA in COS7 cells, suggesting that ARHGAP9 has the GAP activity toward Racl and Rac2 in vivo. We also performed a pull-down assay with EGFP-Rac1, EGFP-Rac2 and EGFP-ARHGAP9- $\Delta$ N-370Ala or EGFP-ARHGAP9- $\Delta \mathrm{N}-370$ Ser. There were no significant differences in the GAP activity between EGFP-ARHGAP9- $\Delta$ N-370Ala and EGFPARHGAP9- $\triangle \mathrm{N}-370$ Ser (Figure 2d).

We next determined whether the PH domain of ARHGAP9 can bind with phosphoinositides because the 370Ala/Ser mutation exists in the $\mathrm{PH}$ domain, which is involved in cell signaling through direct binding to phosphoinositides. ${ }^{26}$ GST-ARHGAP9-PH 370Ala and GST-ARHGAP9-PH 370Ser were subjected to a liposome binding assay (Supplementary Figure 1b). The liposome binding assay showed that GST-ARHGAP9-PH 370Ala bound to phosphatidylinositol 4,5bisphosphate (PtdIns(4,5)P2) and PtdIns(3,4,5)P3 strongly, and that GST-ARHGAP9-PH 370Ser showed almost the same affinities to PtdIns(4,5)P2 and PtdIns(3,4,5)P3 as GST-ARHGAP9-PH 370Ala (Supplementary Figure 1c).

Effect of the Ala370Ser polymorphism in ARHGAP9 on its function Rac1 has a pivotal function in cell migration partly through the regulation of cell to matrix adhesion. This prompted us to examine whether the Ala370Ser polymorphism is related to cell migration. We performed the Boyden chamber assay for Vero cells expressing ARHGAP9 (Figure 3a). The expression of EGFP-ARHGAP9-FL 370Ala and EGFP-ARHGAP9-FL 370Ser both inhibited the migration of Vero cells, but the inhibitory effect of EGFP-ARHGAP9-FL 370Ser was weaker than that of EGFP-ARHGAP9-FL 370Ala, suggesting that the Ala370Ser polymorphism affects the ARHGAP9 activity to suppress cell migration.

ARHGAP9 has an important function in adhesion of cells to the matrix, specifically to fibronectin presumably through the regulation of Racl activity. ${ }^{25}$ Because cell spreading reflects the activity of adhesion of cells to the matrix, the area of HeLa cells and RAW264 cells (mouse leukemic monocytes) expressing ARHGAP9 was measured (Figures 3b, c). The cells expressing EGFP-ARHGAP9-FL 370Ala and EGFP-ARHGAP9-FL 370Ser both spread less than the cells expressing control EGFP-GST. The inhibitory effect of EGFP-ARHGAP9-FL 370Ser was significantly weaker than that of EGFP-ARHGAP9-FL 370Ala, suggesting that the Ala370Ser polymorphism weakens the ARHGAP9 function in cell spreading.

To examine whether the Ala370Ser polymorphism has a function at the stage of cell adhesion to fibronectin, we performed a cell-adhesion assay using Jurkat cells (human T-lymphocyte cells) expressing EGFPGST, EGFP-ARHGAP9-FL 370Ala or EGFP-ARHGAP9-FL 370Ser (Figure 3d). EGFP-ARHGAP9-FL reduced the number of cells attached to fibronectin, and EGFP-ARHGAP9-FL 370Ser was less effective compared with EGFP-ARHGAP9-FL 370Ala in cell attachment as well as cell migration and cell spreading.

We finally measured the production of reactive oxygen species (ROS) in RAW264 cells expressing GFP-ARHGAP9- $\Delta \mathrm{N} 370 \mathrm{Ala}$ or 370Ser, because Rac has a crucial function in the regulation of NADPH oxidase, ${ }^{27,28}$ and ROS and resultant oxidative stress are thought to be involved in the pathogenesis of cardiovascular diseases. Production of ROS in cells expressing ARHGAP9- $\Delta \mathrm{N}$ was slightly decreased, but we could not find a significant difference between ARHGAP9- $\triangle \mathrm{N}$ 370Ala and -370Ser expressing cells (data not shown). This may be due to the low expression levels of ectopically expressed ARHGAP9.

\section{DISCUSSION}

We found here that the Ala370Ser polymorphism in the ARHGAP9 gene is associated with CSA. ARHGAP9 is expressed in hematopoietic cell lines and tissues. ${ }^{25}$ RT-PCR experiments also revealed high levels of ARHGAP9 expression in peripheral leukocytes (Figure 1a). The pull-down assay showed that EGFP-ARHGAP9-GAP mainly inactivated EGFP-Rac1 and EGFP-Rac2 in vivo (Figures $2 \mathrm{~b}, \mathrm{c}$ ). Rac1 is ubiquitously expressed, whereas Rac2 is specially expressed in hematopoietic cells. These results indicate that ARHGAP9 negatively regulates Rac1 and Rac2 activities as RacGAP in hematopoietic cells.

We also examined whether this polymorphism affects the ARHGAP9 functions. We found that the polymorphism did not influence the mRNA levels of ARHGAP9 and ectopically expressed ARHGAP9 proteins, or the binding activity to phosphoinositides. We performed several pull-down assays with various cell lines under stimuli such as growth factors and fibronectin to examine whether the ARHGAP9 370Ser mutation directly reduces RacGAP activity in vivo, but we did not clearly confirm that the ARHGAP9 370Ser mutation influences RacGAP activity. However, the results of the spreading assay and cell migration assay seem to support the notion that the ARHGAP9 370 Ser mutation decreases RacGAP activity in vivo. We suppose that the localized regulation of ARHGAP9 activity would be important for spreading and migration. If the ARHGAP9 370Ser mutation affects RacGAP activity only at the local area, such as plasma membrane, it is probably difficult to reveal the difference of GAP activity between ARHGAP9 370Ala and ARHGAP 370Ser by a pull-down assay, because the assay reflects the GAP activity in the whole cell. Thus, it is likely that the ARHGAP9 370Ser mutation lowers its RacGAP activity.

Endothelial dysfunction is characterized by a shift in the actions of the endothelium toward reduced vasodilation, ${ }^{8}$ and is a critical factor in the pathogenesis of CSA. ${ }^{3,5}$ Endothelial dysfunction is caused by risk factors related to atherosclerosis, such as hyperlipidemia, smoking and diabetes. ${ }^{29}$ Although the causal mechanism of endothelial dysfunction remains elusive, available evidence suggests that chronic low-grade inflammation in arteries including the endothelium has an important function in the induction of endothelial dysfunction with coronary artery spasm and atherosclerotic changes. ${ }^{10,30,31}$

The multistep paradigm occurs when leukocytes cross the endothelium to enter tissues in inflammatory and immune responses. ${ }^{32}$ The first step is the rolling of the leukocytes over the endothelium. This initial contact allows leukocytes to spread and adhere to the endothelium. The arrested leukocytes crawl to search for a gateway to cross the endothelium and finally migrate to the underlying tissue. Rac regulates the adhesion, spreading and migration of neutrophils and macrophages. ${ }^{33,34}$ Rac also regulates ROS generation, which has celldamaging effects. ${ }^{27,28}$ We found here that EGFP-ARHGAP9 reduced the migration of Vero cells, the spreading of HeLa cells and RAW264 cells, and the cell adhesion of Jurkat cells, suggesting that ARHGAP9 controls the infiltration of leukocytes from the blood across the vascular endothelium into tissues. The EGFP-ARHGAP9 370Ser mutation suppressed the reduction of cell migration, cell spreading and cell attachment (Figure 3). These results raise the possibility that leukocytes and monocytes with the ARHGAP9 370Ser mutation cause an inflammation of the vascular endothelium more severely than 


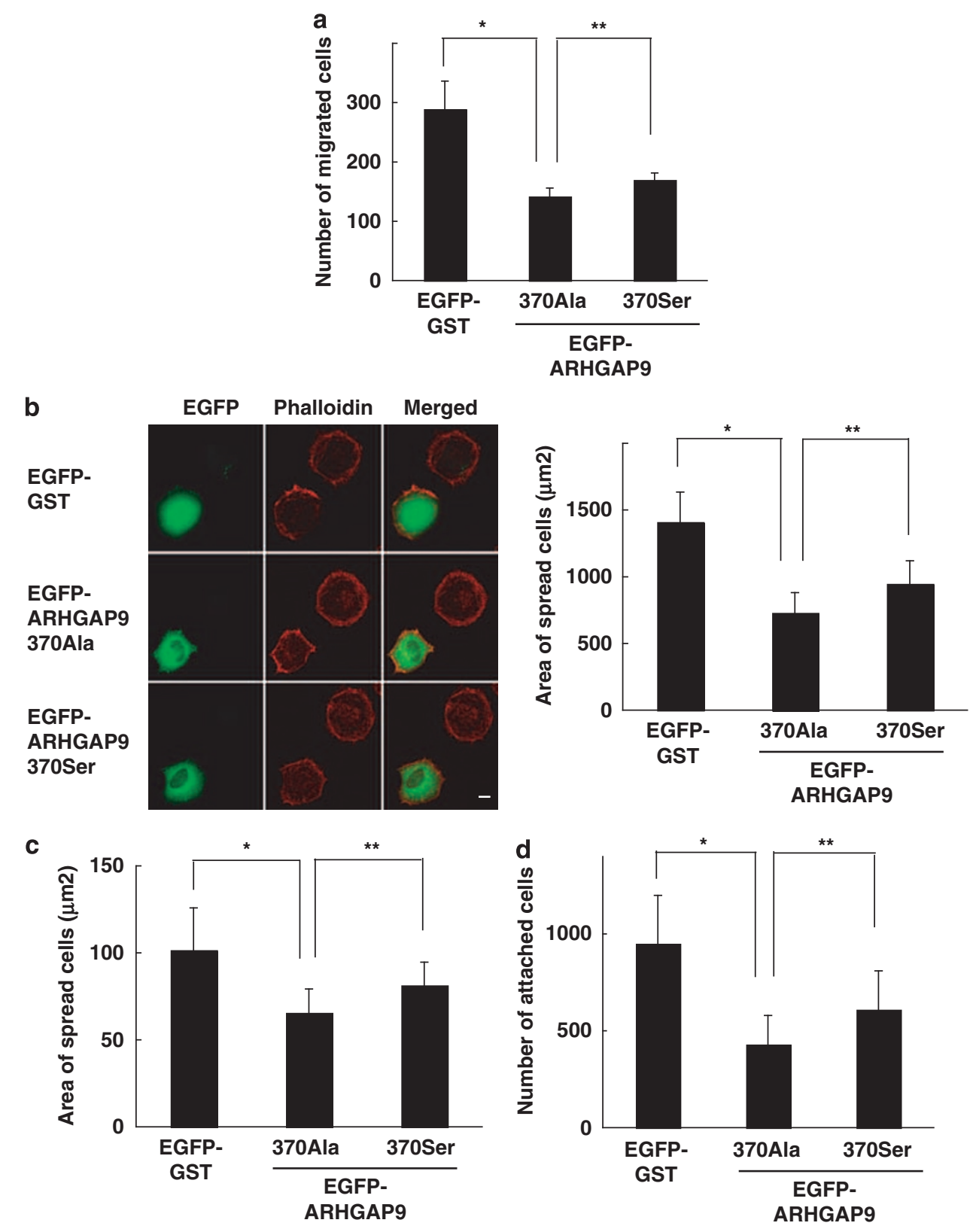

Figure 3 Effects of ARHGAP9 370Ala and 370Ser on cell adhesion and cell migration. (a) Effect of ARHGAP9-FL 370Ala and 370Ser on cell migration in a Boyden chamber assay. Vero cells were transfected with the indicated plasmids and subjected to a Boyden chamber assay. The membrane of the lower side of the upper chamber was coated with fibronectin. Data are expressed as mean \pm s.d. (b and $\mathbf{c}$ ) Effect of ARHGAP9-FL 370Ala and 370Ser on spreading HeLa cells (b) and RAW264 cells (c). HeLa and RAW264 cells were transfected with the indicated plasmids and the area of spread cells was measured ( $n>200$ in each experiment). Scale bar, $10 \mu \mathrm{m}$. (d) Effect of ARHGAP9-FL 370Ala and 370Ser on cell attachment of Jurkat cells. Jurkat cells were transfected with EGFP-GST or EGFP-ARHGAP9-FL. GFP-positive cells (5000) were plated on coverslips precoated with fibronectin. After 30-min incubation, cells were fixed and the number of GFP-positive cells was counted. Asterisks indicate significant differences $\left({ }^{*} P<0.005\right.$; $\left.{ }^{* *} P<0.01\right)$ from the value of EGFP-GST or EGFP-ARHGAP9-FL 370Ala.

those with ARHGAP9 370Ala. As a result, inflammatory endothelial dysfunction probably occurs more frequently in subjects with ARHGAP9 370Ser than those with ARHGAP9 370Ala. We could not detect a significant difference in the generation of ROS between ARHGAP9 370Ser- and ARHGAP9 370Ala-expressing cells under our experimental conditions. Nonetheless, it is still possible that this polymorphism results in a slightly different ROS production, which synergistically influences the endothelial dysfunction with differences in cell adhesion and migration.
As for the relation of small GTPases to the incidence of CSA, Rho/ Rho-kinase signaling has mainly been proposed for the $\mathrm{Ca}^{2+}$-independent contraction of vascular smooth muscle in CSA. In this study, we found that ARHGAP9 is a genetic risk factor for CSA. Our results reveal two new critical phases: (1) not only small GTPases and their effectors but also the regulators of small GTPases have relevance to the incidence of CSA, and (2) small GTPases signaling probably contributes to endothelial dysfunction as well as contraction of vascular smooth muscle in patients with CSA. 


\section{ACKNOWLEDGEMENTS}

This work was supported in part by Grants-in-Aid for Creative Science Research and a Grant-in-Aid for Scientific Research from the Ministry of Education, Culture, Sports, Science and Technology of Japan. We thank all the individuals who participated in the study.

1 Oliva, P. B., Potts, D. E. \& Pluss, R. G. Coronary arterial spasm in Prinzmetal angina. Documentation by coronary arteriography. New Engl. J. Med. 288, 745-751 (1973).

2 Ong, P., Athanasiadis, A., Hill, S., Vogelsberg, H., Voehringer, M. \& Sechtem, U. Coronary artery spasm as a frequent cause of acute coronary syndrome: the CASPAR (Coronary Artery Spasm in Patients With Acute Coronary Syndrome) Study. J. Am. Coll. Cardiol. 52, 523-527 (2008)

3 Yasue, $H$. Nakagawa, $H$., Itoh, T., Harada, E. \& Mizuno, $Y$ Coronary artery spasmclinical features, diagnosis, pathogenesis, and treatment. J. Cardiol. 51, 2-17 (2008).

4 Miyao, Y., Kugiyama, K., Kawano, H., Motoyama, T., Ogawa, H., Yoshimura, M. et al. Diffuse intimal thickening of coronary arteries in patients with coronary spastic angina. J. Am. Coll. Cardiol. 36, 432-437 (2000).

5 Kawano, H. \& Ogawa, H. Endothelial function and coronary spastic angina. Inter. Med. 44, 91-99 (2005)

6 Furchgott, R. F. \& Zawadzki, J. V. The obligatory role of endothelial cells in the relaxation of arterial smooth muscle by acetylcholine. Nature 288, 373-376 (1980).

7 Moncada, S., Palmer, R. M. \& Higgs, E. A. Nitric oxide: physiology, pathophysiology, and pharmacology. Pharmacol. Rev. 43, 109-142 (1991).

8 Endemann, D. H. \& Schiffrin, E. L. Endothelial dysfunction. J. Am. Soc. Nephrol. 15, 1983-1992 (2004).

9 Kaikita, K., Ogawa, H., Yasue, H., Sakamoto, T., Suefuji, H., Sumida, H. et al. Soluble $\mathrm{P}$-selectin is released into the coronary circulation after coronary spasm. Circulation $\mathbf{9 2 ,}$ 1726-1730 (1995).

10 Hung, M. J., Cherng, W. J., Yang, N. I., Cheng, C. W. \& Li, L. F. Relation of highsensitivity C-reactive protein level with coronary vasospastic angina pectoris in patients without hemodynamically significant coronary artery disease. Am. J. Cardiol. 96, 1484-1490 (2005).

11 Miwa, K., Igawa, A. \& Inoue, H. Soluble E-selectin, ICAM-1 and VCAM-1 levels in systemic and coronary circulation in patients with variant angina. Cardiovasc. Res. 36, 37-44 (1997).

12 Pristipino, C., Beltrame, J. F., Finocchiaro, M. L., Hattori, R., Fujita, M., Mongiardo, R. et al. Major racial differences in coronary constrictor response between Japanese and Caucasians with recent myocardial infarction. Circulation 101, 1102-1108 (2000).

13 Baba, S., Ozawa, H., Sakai, Y., Terao, A., Konishi, M. \& Tatara, K. Heart disease death in a Japanese urban area evaluated by clinical and police records. Circulation 89, 109 115 (1994).

14 Miwa, K., Fujita, M. \& Sasayama, S. Recent insights into the mechanisms, predisposing factors, and racial differences of coronary vasospasm. Heart Vessels 20, 1-7 (2005).
15 Kimura, K., Ito, M., Amano, M., Chihara, K., Fukata, Y., Nakafuku, M. et al. Regulation of myosin phosphatase by Rho and Rho-associated kinase (Rho-kinase). Science 273, 245-248 (1996).

16 Fukata, Y., Amano, M. \& Kaibuchi, K. Rho-Rho-kinase pathway in smooth muscle contraction and cytoskeletal reorganization of non-muscle cells. Trends Pharmacol. Sci. 22, 32-39 (2001).

17 Somlyo, A. P. \& Somlyo, A. V. Ca2+ sensitivity of smooth muscle and nonmuscle myosin II: modulated by G proteins, kinases, and myosin phosphatase. Physiol. Rev. 83, 1325-1358 (2003).

18 Raftopoulou, M. \& Hall, A. Cell migration: Rho GTPases lead the way. Dev. Biol. 265 23-32 (2004).

19 Rolfe, B. E., Worth, N. F., World, C. J., Campbell, J. H. \& Campbell, G. R. Rho and vascular disease. Atherosclerosis 183, 1-16 (2005).

20 Jaffe, A. B. \& Hall, A. Rho GTPases: biochemistry and biology. Annu. Rev. Cell Dev. Biol. 21, 247-269 (2005).

21 Murase, Y., Yamada, Y., Hirashiki, A., Ichihara, S., Kanda, H., Watarai, M. et al. Genetic risk and gene-environment interaction in coronary artery spasm in Japanese men and women. Eur. Heart J. 25, 970-977 (2004).

22 Yamada, Y., Izawa, H., Ichihara, S., Takatsu, F., Ishihara, H. et al. Prediction of the risk of myocardial infarction from polymorphisms in candidate genes. New Engl. J. Med. 347, 1916-1923 (2002)

23 Kondo, K., Shintani, S., Shibata, R., Murakami, H., Murakami, R., Imaizumi, M. et al. Implantation of adipose-derived regenerative cells enhances ischemia-induced angiogenesis. Arterioscler. Thromb. Vasc. Biol. 29, 61-66 (2009).

24 Nakayama, M., Amano, M., Katsumi, A., Kaneko, T., Kawabata, S., Takefuji, M. et al. Rho-kinase and myosin II activities are required for cell type and environment specific migration. Genes Cells 10, 107-117 (2005).

25 Furukawa, Y., Kawasoe, T., Daigo, Y., Nishiwaki, T., Ishiguro, H., Takahashi, M. et al. Isolation of a novel human gene, ARHGAP9, encoding a rho-GTPase activating protein. Biochem. Biophys. Res. Commun. 284, 643-649 (2001).

26 Itoh, T. \& Takenawa, T. Phosphoinositide-binding domains: functional units for temporal and spatial regulation of intracellular signalling. Cell. Signal. 14, 733-743 (2002).

27 Greomping, Y. \& Rittinger, K. Activation and assembly of the NADPH oxidase: a structural perspective. Biochem. J. 386, 401-416 (2005).

28 Bokoch, G. M. Regulation of innate immunity by Rho GTPases. Trends Cell Biol. 15, 163-171 (2005).

29 Bonetti, P. O., Lerman, L. O. \& Lerman, A. Endothelial dysfunction: a marker of atherosclerotic risk. Arterioscler. Thromb. Vasc. Biol. 23, 168-175 (2003).

30 Danesh, J., Wheeler, J. G., Hirschfield, G. M., Eda, S., Eiriksdottir, G. \& Rumley, A. et al. C-reactive protein and other circulating markers of inflammation in the prediction of coronary heart disease. New Engl. J. Med. 350, 1387-1397 (2004).

31 Jarvisalo, M. J., Juonala, M. \& Raitakari, O. T. Assessment of inflammatory markers and endothelial function. Curr. Opin. Clin. Nutr. Metab. Care 9, 547-552 (2006).

32 Butcher, E. C. Leukocyte-endothelial cell recognition: three (or more) steps to specificity and diversity. Cell 67, 1033-1036 (1991).

33 Jones, G. E., Allen, W. E. \& Ridley, A. J. The Rho GTPases in macrophage motility and chemotaxis. Cell Adhes. Commun. 6, 237-245 (1998).

34 Gardiner, E. M., Pestonjamasp, K. N., Bohl, B. P., Chamberlain, C., Hahn, K. M. \& Bokoch, G. M. Spatial and temporal analysis of Rac activation during live neutrophil chemotaxis. Curr. Biol. 12, 2029-2034 (2002).

Supplementary Information accompanies the paper on Journal of Human Genetics website (http://www.nature.com/jhg) 